\title{
Electrical characteristic of new calculation in sub-transmission line with simulation
}

\author{
S. Firouzifar E.Dahlquist \\ Malardalen University, Vasteras, Sweden, sfirouzifar@yahoo.com
}

\begin{abstract}
Important developments for controlling over voltage and conductor resonances in recent decades has given the possibility of reducing the phase distances in vast range. By this optimization can be made of some of the electric parameters in comparison with normal distribution lines, increasing the capacity in energy transmission. The reduction of the reactance of the line, increasing capacitance, increasing serge impedance loading (SIL) and reduction or increase of some of the other electrical parameters such as the geometric mean distance (GMD), will increase the capacity of this kind of modern distribution lines in comparison to normal distribution lines. By doing this, optimization can be made for different parts of the net. In this paper, a program in windows using Delphi software is presented for this optimization. The electrical specifications of modern and normal distribution lines are compared.
\end{abstract}

Keywords: SIL-GMD surge impedance, modern line, sub-transmission line

\section{Nomenclature}

$\begin{array}{ll}G D M & \text { Geometric Mean Distance } \\ G M R & \text { Geometric Mean Radius } \\ X_{I} & \text { Inductive reactance of distribution line } \\ X_{c} & \text { Capacitance reactance of distribution line } \\ S I L & \text { Serge Impedance Loading } \\ L & \text { Inductance } \\ C & \text { Capacitance } \\ Z & \text { Serge Impedance } \\ d s & \text { Distance between sub- conductors } \\ n s & \text { Number of extraneous } \\ r & \text { Radius in centimeter } \\ \mu & \text { Respective permeability } \\ \lambda & \text { Wave length of line } \\ U & \text { Velocity of wave propagation }\end{array}$

\section{Introduction}

If we want to transmit energy in alternating current with acceptable efficiency, we must provide the minimum drop of voltage and energy. Alternating current is accompanied with reactive power and the transmission capacity of the line is determined by this. For suitable operation and using the maximum transmission capacity of the line, the reactive power should be minimized for any current load. If we provide these conditions, we can transmit energy without any loss due to reactive and capacitive characteristic. We can reduce the reactive power of the line by balancing between reactive power of line and additional equipment like capacitors or reactors. The effect cost for these equipment can be predicted, respectively. Transmission line design is discussed in (Clerci and Landonin, 1991; Heidari and Heidari, 2002; Doss, 2002). More details of technology is available e.g. in (Alcola Conduc tor Accessories, 2003; ACCR, 2003; 3M, 2003).

Another way to reduce the reactive power of lines is to balance between the inductive and capacitive reactive power of the line as such, whereby the total reactive power of the line will be minimum and about zero. The balancing between inductive reactive power and capacitive reactive power, can be possible by selecting the suitable physical shape of pilars and suitable placement of conductors. In this way, we don't need additional equipment such as classical lines. For modern compared to normal lines, a basic review in calculation and reduction of phase distance is required. Gas insulated transmission lines (GIL) are discussed in (Koch and Schuette, 1997; Hiller and Koch, 1997; Hiller and Koch, 1998a; Chakir and Koch, 2001; Chakir and Koch, 2002) and their applications in metropolitan areas (Hiller and Koch, 1998b), Heningen et al. (2000) focus on long-time electrical and mechanical behavior of GILs. Full scale tests are presented in (CRIEPI).

The advantage of this process is increasing the capacity, reducing the passing band width and reducing the line cost. The research shows that reducing the geometric mean distance (GMD) in a wide range is possible. (Markus et al., 2000; Inventions and Innovation, 1999).

\section{Designing modern lines}

\subsection{Sub-transmission and distribution voltages}

In Iran, the 10-20 kV voltages are considered as distribution voltages and the $63-132 \mathrm{kV}$ voltages as sub transmission voltages. The length of these lines is several tens multiplied by the length of transmission lines. Distribution and sub transmission nets provide the required energy for consumers. For this reason all of the 
cities, province centers, industrial centers and productive centers, agricultural and animals' installations, are connected to distribution $20 \mathrm{kV}$ or subtransmission $63 \mathrm{kV}$ nets. For this voltage range, the classic approach is to not use groups of conductors. Lack of the conductor group and incremental capacitive properties, causes problems in the designing of modern lines. The other property of distribution lines is not the use of tall towers with wooden or concrete legs but our main discussion in this article, are the GMD variations for increasing natural power or the serge impedance loading (SIL) of the line (Alexandrov and Heidari; Koch and Schuette, 1997).

\subsection{Geometric mean distance of phases}

One of the most important parameters for defining the inductance and capacitance of distribution lines is the distance between line phases. By assuming transposed line on the way of it we use, the GMD to evaluate the transmission line parameters. As the distance between conductors in the tower arrangement is defined, the expression (1) is achieved.

$$
X_{l}=0.1447 \times \log \left(\frac{G M D}{G M R}\right)
$$

where the quantity of GMR is the geometric mean radius of filled cylindrical conductors. Normally, if we replace the phases in towers, even for a specified distribution line, the GMD can have different quantities. As the erected shape of modern lines in the appendix of this article shows, according to the phase's arrangement, the GMD varies in a wide range. So, the electrical parameters of distribution lines have vast varieties according to the conductors' arrangement.

\subsection{Inductive reactance}

Inductive reactance is one of the most important parameters in voltage loss, stability limit and active and reactive losses and distribution line capacity too. Normally if we reduce the inductive reactance, we get some benefits for the lines. To calculate the inductance in a three-phase distribution line, we can use (1) for conductors of one kind that we get from

$$
\mu / 4 G M R=r . e
$$

where $r$ is the radius in centimeter and $\mu$ is the respective permeability of conductor that for copper and aluminum is equal to one. Most of the distribution lines are filamentary, and in some cases these filaments are of different kind like (ACSR). So the real quantity of GMR is a little different from the formula. By replacing the quantity of $\mu$ in (2), we can calculate GMR by

$$
G M R=0.7788 \times r
$$

Above formulas are related to simple distribution lines with one conductor in every phase. If distribution lines are installed in the form of bundles (some conductors in each phase) the quantity of GMR must be corrected as

$$
\begin{aligned}
& G M R_{b}=\sqrt[n s]{n s \times G M R \times A^{n s-1}} \\
& \left.A=\frac{d s}{2 \times \sin \frac{\pi}{n s}}\right)
\end{aligned}
$$

In above formulas $n s$ is the number of extraneous conductors in each phase, $d s$ is the distance between extraneous conductors in centimeter and $A$ is area of conductor. Above formulas show that if we reduce the phase distance, the quantity of line will decrease the variations of inductive reactance of lines in the GMD for several conductors compared to only one. In (3), the variation of inductive reactance of line according to the number of conductors in each phase for (curlew) conductors has been shown. It is shown in the fig that, if we compare the inductive reactance of simple and bundle lines in some cases, the inductive reactive reactance of simple modern lines (one conductor in each phase) is nearly equal to normal double bundle lines.

\subsection{Capacitive reactance}

Distribution lines are the consumers of reactive power and because of capacitive property, phases produce reactive power too. In a three-phase distribution line, the capacitance reactance $X_{c}$ of the distribution line is as

$$
X_{C}=0.1318 \times \log \frac{G M D}{r . r_{b}}
$$

where $r_{b}$ is the radius of the group of conductors in centimeter. Other parameters are corresponding to former definitions. By designing modern distribution lines that have less phase distance than normal lines, the susceptance of line will increase too. For reduction of the amount of capacitive reactance or increasing the susceptance of line, we can use more numbers of extraneous conductors in each phase.

\subsection{Surge impedance}

Surge or natural impedance in distribution lines is the amount of consuming impedance when the generative and consuming VAR are equal

$$
\begin{aligned}
& Q_{C}=C W V^{2} \\
& Q_{L}=L W V^{2}
\end{aligned}
$$

when the generative VAR $\left(Q_{C}\right)$ and consuming VAR $\left(Q_{L}\right)$ of distribution lines are equal $(\mathrm{QC}=\mathrm{QL})$ we have

$$
\left(\frac{V}{I}\right)^{2}=\frac{L \cdot W}{C \cdot W}
$$




$$
\begin{aligned}
& Z_{S}=\frac{V}{I} \\
& Z_{S}=\sqrt{L / C}
\end{aligned}
$$

\subsection{Natural power of line}

The other factor that is important in distribution lines is the natural power of line (surge impedance loading). This is the power needed to load with pure resistance and equal surge impedance. If the transmission power of the line is equal to the natural power of the line, the voltages at both ends of the line are nearly equal. So the power can be uses as an important index in loading distribution lines. The amount of natural power of distribution line depends on the amount of reactance and susceptance of the line. We can use the following formulas

$$
\begin{aligned}
& S I L=\frac{U^{2}}{Z_{s}} \\
& S I L=\frac{U^{2}}{\sqrt{L / C}}
\end{aligned}
$$

to calculate them (Sganzerla et al., 1996; Alexandrov and Heidari; Heidari, 1995: Heidari and Alexandrov, 1996).

$$
\text { As you see in }
$$

$$
\mathrm{V}=1 / \sqrt{l . c}
$$

this power is a function of the inductive and capacitive reactances of the line. As much as is reduced from reactance (or is increased to the capacitance of line), so much the surge impedance loading of the line will increase. Modern or bundle distribution lines that have low inductive reactance have more natural power inductance multiplied by the capacitance of the line. This is a function of the voltage of electromagnetic waves, that is expressed as below

As seen in (14), $v$ or the velocity of electromagnetic waves is nearly constant. The amount of inductance multiplied by capacitance are constant too. It means that that whatever reactance decreases, capacitance increases and leads to an increase in the quality of distribution line. By combining formulas (12), (13) and (14), we can show the natural power of the line is a function of line voltage, the velocity of electromagnetic waves and inductive and/or capacitive reactance of the line:

$$
\begin{aligned}
& S I L=C \cdot V \cdot U^{2} \\
& S I L=\frac{U^{2}}{L \cdot V}
\end{aligned}
$$

These formulas show that, because of constant amounts of a distribution line, natural power is a function of inductive or capacitive reactance of the lines. In other words, increasing or decreasing the amount of inductive and/or capacitive reactance of a line directly affects the natural power of the line.

\section{Modern means voltage line}

The $20 \mathrm{kV}$ lines as distribution lines are usually forecasted and built as one or two circuits on cement or wooden tower (Figure 1). We need a distance about 1.52 meters between the phases, according to the tower distance or span. This decreases the capacitive property, but also increases the required right-of-way in the transmission line. In modern lines, the distance between phases in tower place and along the span is decreased by $0.4-0.5$ meters. So the capacitive property of the line increases about $20-30 \%$. By a suitable design of line, forecasting isolated insulators and using polymer insulators and assuming three phases in three vertexes of a triangle, the phase distance is limited to 0.5 meter. (Heidari and Alexandrov, 1996)

According to phase distance the amount of surge impedance in existed lines is $370 \Omega$ and in modern lines $250 \Omega$. For case one, natural power of the line is

$$
S I L=\frac{U^{2}}{Z_{S}}=1.1 \mathrm{MW}
$$

For modern line by installing three similar phases we will have

$$
S I L=\frac{U^{2}}{Z_{s}}=1.8 \mathrm{MW}
$$

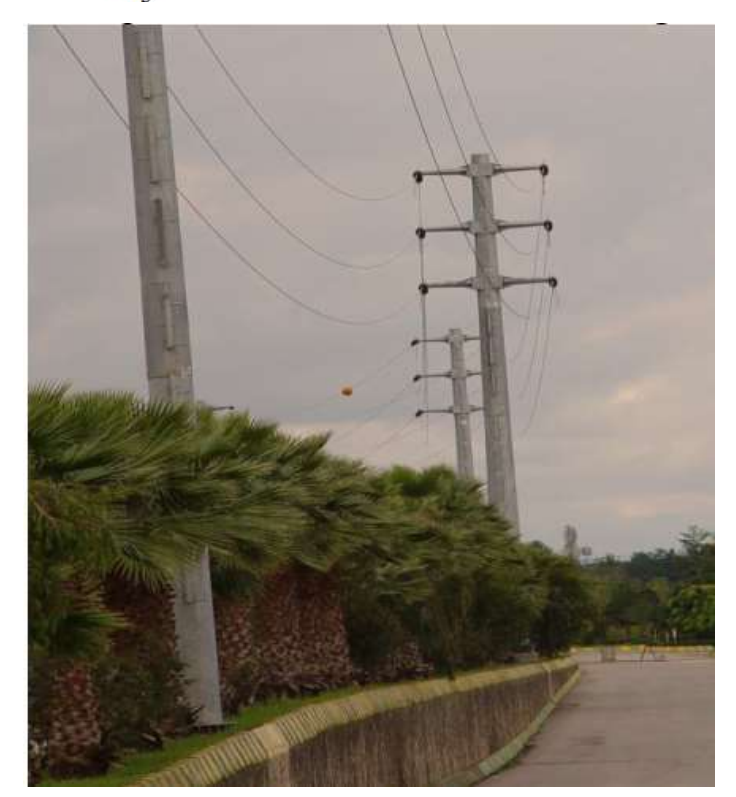

Figure 1. Voltage line.

As you see, the SIL or the natural power of $20 \mathrm{kV}$ line increases about $0.7 \mathrm{MW}$. The reactive power for two kinds of above lines with $15 \mathrm{MW}$ nominal power and 20 $\mathrm{km}$ length is calculated as below. 
A) Line with GMD = 1.5 (existing line)

$$
\begin{aligned}
& Q_{1}=\omega C U^{2}-\omega L I^{2}=\omega C U^{2}\left(1-\frac{L I^{2}}{C U^{2}}\right) \\
& =\omega C U^{2}\left(1-\frac{U^{2} I^{2}}{\left(\frac{U^{2}}{\sqrt{L / C}}\right)^{2}}\right) \\
& =S I L \cdot \lambda\left[1-\left[\frac{P}{S I L}\right]^{2}\right]=1.15 M V A R
\end{aligned}
$$

B) Line with $\mathrm{GMD}=0.5$ (modern line)

$$
Q_{L}=S I L \cdot \lambda\left[1-\left[\frac{P}{S I L}\right]^{2}\right]=0.65 M V A R
$$

where $\lambda$ is the wave length of line and $v$ is the velocity of wave propagation $=300,000 \mathrm{~km} / \mathrm{sec}$.

As is seen, the consumed reactive power of the line has decreased about a half. As the load of $15 \mathrm{MW}$, was an industrial load and is maintained continuously and stable in 24 hours, decreasing reactive power to half, will accompany considerable economy from decreasing losses. By providing software we can do the calculations by computer easier and the results are analyzed faster and in for a wide range of data.

\section{Program provided in Delphi}

This program can do the electrical calculations of the line from the formulas in presented in this article. This capability means, according to the conditions, we can find the best situation by different inputs. The reports contain all electrical information of the line. As it was said in Section 2.7 about the GMD changes, the program can handle it.

By drawing two charts of the GMD changes according to SIL and P/SIL, we can observe the charts of changes by decreasing and increasing GMD and a boundle line or multicircuit lines that we show as examples. The page of reports contains all electrical data of the line. It is opened in the page of input data by specifications that are shown in Figure 2 and pressing the key of doing calculations.

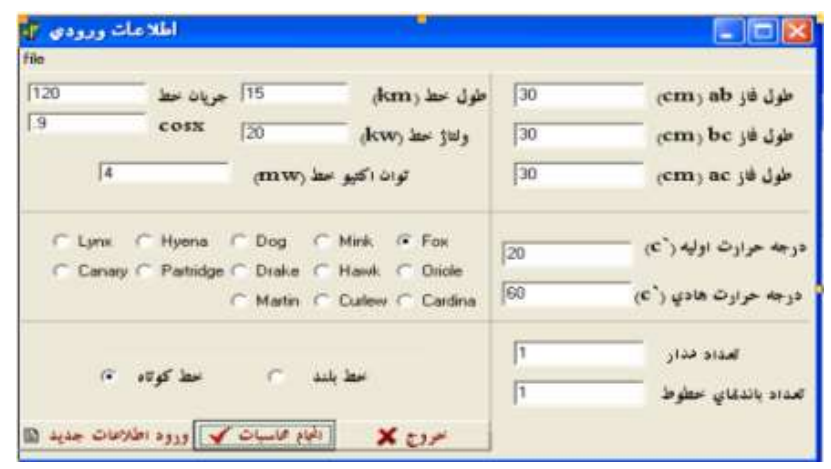

Figure 2. Face plate for input data.

As you see in Figure 2, the phase distance of line is specified by assuming that the line is modern, the phase distances equal. The ambient or primary temperature and the temperature of the under load conductor are considered too.

The circuits' numbers of line can calculate up to three. As you see in (4) and (5), if the number of line bundles is one, the number of line for $\mathrm{n}$ boundles can be calculated. If the number of line boundle is one, the other data of the line like the line length, line current, line voltage, $\cos \varphi$ and the active power of line for accurate calculations are required. If the line is long, $\pi$ model can be used for that.

The output data of the calculations are seen in Figure 3. This has all the required electrical data for line design as it is shown with the data in Figure 3: the losses of the line are 1.04 .

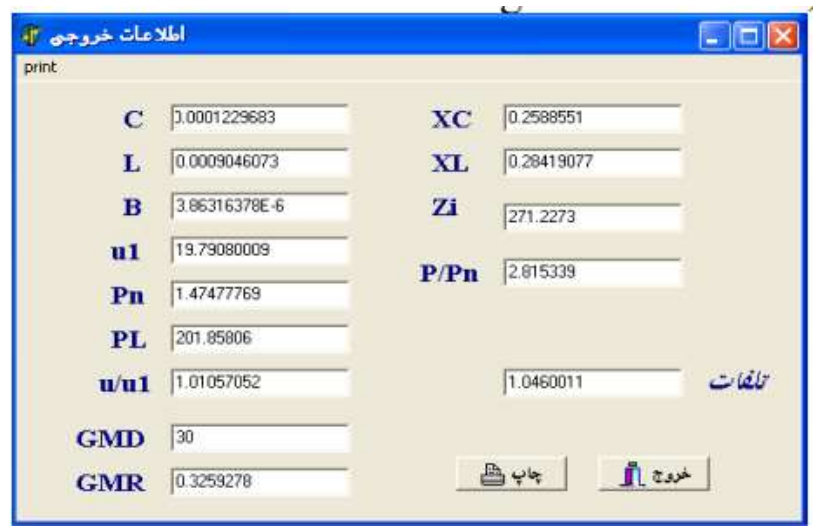

Figure 3. Output data from the calculations.

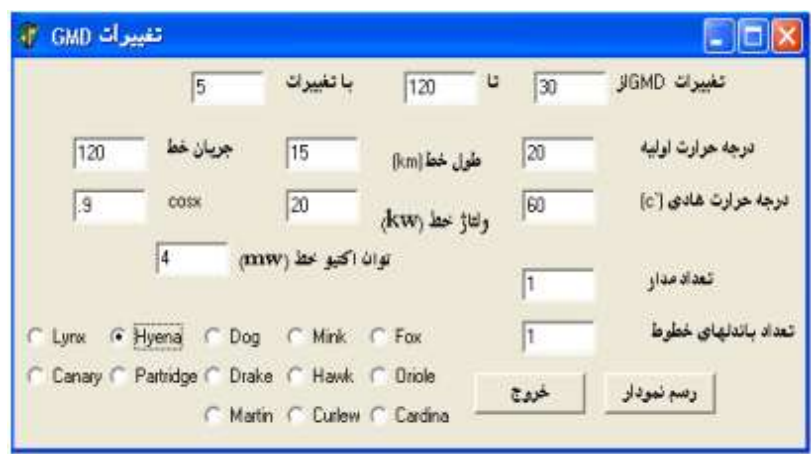

Figure 4. Table with relationship between changes in GMD with different kind of conductor.

In Figure 4, the change in the SIL can be seen by changing the GMD and it is concluded that by increasing the GMD means increase in the phase distance and the SIL, the natural power of the line decreases (Figure 5). 


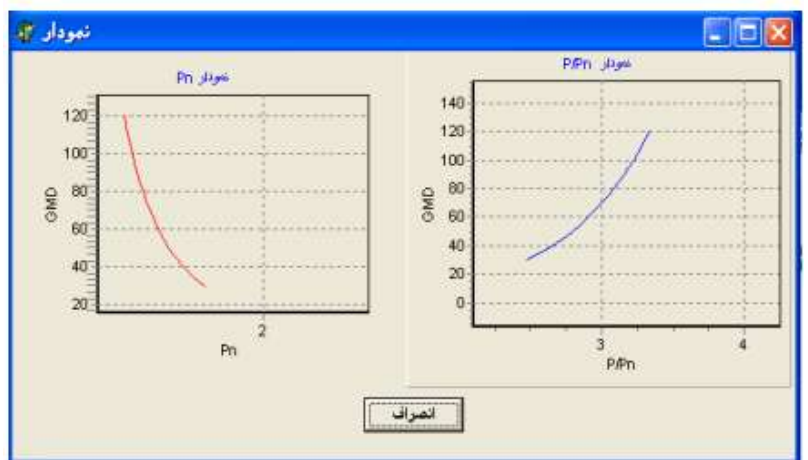

Figure 5. Curve GMD vs. SIL and GMD vs. P/SIL.

\subsection{The ratio of transmitted power to natural power of line P/SIL}

When the induced reactive power of a line is minimum, the ideal ratio of $\mathrm{P} / \mathrm{SIL}$ is equal to one. To reach this amount $\mathrm{P} / \mathrm{SIL}=1$ we can not reduce $\mathrm{P}$, because decreasing $\mathrm{P}$ and conductor's section is not economic. However, we can increase SIL and this is achieved by decreasing impedance $Z_{s}$ that increases the capacitive property of the line as below:

$$
S I L=\frac{U^{2}}{Z s} \longrightarrow Z s=\sqrt{L / C}=\frac{1}{X . C} \mathrm{~V}
$$

The propagation velocity of wave is equal to

$$
\frac{1}{\sqrt{L . C}}
$$

\subsection{Surveying the GMD changes and its effects on the ratio of transmitted power to Surge Impedance Loading.}

By changes in GMD, we can see the changes in $\mathrm{C}$, $\mathrm{L}$, $\mathrm{Zs}$, and at last SIL that is the natural power of the line. The purpose of this program is decreasing the inductance of line (L). A comparison between the conventional and compact lines with consideration (GMD) is presented in the appendix, the difference between a normal $20 \mathrm{kV}$ line and a modern $20 \mathrm{kV}$ line shows that the natural power in modern lines is more than in normal lines. By increasing the natural power according to decreasing GMD and changes in phase arrangement, power is increased. Increasing transmitted power will decrease the ratio of transmitted power to the natural power of line SIL (Figure 5). By decreasing the GMD, this ratio is near to one now. In $20 \mathrm{kV}$ lines, the ratio of $\mathrm{P} / \mathrm{SIL}$ is more than three, but we must make it near to 1 by changing the phase arrangement.

The provided software has this capability to decide how much we should change the GMD, and to find the optimum point of the GMD for every voltage. For example, the best status of phase's arrangement in 20 and $63,33 \mathrm{kV}$ voltages can be defined. At the same time, the difference between two circuits and two boundles or three circuits, can be specified pluralization and deduction: don't need to develop a bay at $63,20 \mathrm{kV}$ stations.

\section{Conclusions}

According to two basic theories in modern transmitting energy:

- For transmitting energy in modern lines, it is better to have two or three circuit lines as more energy can be transmitted.

- In the second theory, its better to transmit energy in two or more boundless, because in this situation we can in fact transmit more energy and don't need to pay anything for the development of the station bay.

However, regard to the above reasons, we have a new suggestion in this article. By using Delphi software and several experiments we concluded that for transmitting energy, if we want to use the number of circuits, construct the transmission lines with two circuits, and if we require to transmit more energy it is better to construct a modern line with three or four boundless, instead of three circuit line, because the natural power of a three-boundle line is more than a three-circuit line.

For increasing the SIL, the natural power, of 20, 33 and $63 \mathrm{kV}$ lines, the major solution is an increasing capacitive property by nearing three-phase conductors to each other and providing the minimum distance of the phase to phase isolation. By this way, we conclude the following:

1) Increasing the capacitive property of line.

2) Reducing voltage and power loss.

3) Reducing the losses of line.

4) Reducing the surge impedance.

5) Reducing the inductive inductance.

6) Increasing the stability of line.

Our research shows that it is possible to reduce GMD in a wide range. By modern lines in 20,33 and $63 \mathrm{kV}$ voltages, we can reduce the GMD triply.

\section{References}

ACCR. Power Conductor Accelerated Testing Facility (PCAT), ACCR Technology Description, November 2003.

Alcola Conductor Accessories. Transmission Accessories, 2003.

G. N. Alexandrov and H. Heidari. Equalization of overhead Ch. RI, "Heida" Increasing Lind Voltage.

Aluminum Conductor Composite Reinforced, Technical Notebook, 3M, March 2003.

A. Chakir and H. Koch. Thermal Calculation for Buried Gas -Insulated Transmission Lines (GIL) and XLPE-Cale, In IEEE Winter Power Meeting 2001, Columbus, 2001.

A. Chakir and H. Koch. Long Term Test of Buried Gas Insulated Transmission Lines (GIL). In IEEEWPM 2002 New York, 2002.

A. Clerci and M. Landonio. EHV Compact Lines - a new solution. In CIGRE, 1991. 
CRIEPI, Report: Development of Make 66 to $154 \mathrm{kV}$ Overhead Compact Transmission Lines (Part7) - Study on Mechanical Stresses to $154 \mathrm{kV}$ Insulation Arms on FullScale Actual Test. CRIEPI Rep. W95037

Dennis Doss. Double your transmission capacity without changing existing towers. In Electric light 7 and Cable, August 2002.

Gh. Heidari. Equalization of overhead transmission line capacity different classes of voltage. In PSC 95, Tehran, Nov. 1995.

Gh. Heidari and G. N. Alexandrov, Increasing line voltage or sub conductors number each phases. In CIGRE, Paris, France, Sept. 1996.

Gh. Heidari and Maziar Heidari. Effect of land price on transmission line design. In CIGRE, Sept. 2002.

C. G. Heningen, G. Kaul, H. Koch, A. Scheuette, and R.Plath. Electrical and Mechanical Long-Time Behaviour of Gas Insulated Transmission Lines. In CIGRE, 08/2000.

T. Hiller and H. Koch. Gas Insulated Transmission Lines (GIL): proven technology with new performance. In Xiamen Switchgear Seminar, China, 1997

T. Hiller and H. Koch. Gas-Insulated Transmission Lines for high power transmission over long distances. In EMPT, Singapore, 1998a.

T. Hiller and H. Koch. Gas -insulated Transmission Lines (GIL): A solution for the power supply of metropolitan areas. In CEPSI, Thailand, 1998b.

H. Koch and A. Schuette. Gas Insulated Transmission over long distances. In EPSR, Hong Kong, 1997.

Inventions \& Innovations. Development of a CompositeReinforced Aluminum Conductor, Project Fact Sheet, 1999.

H. B. Markus, P. Vestner, Diego F. Sologuren-Sanchez, Bodo A. Bruhl, and Paul F. Coventry. Dimensioning criteria and test result for a polymer enclosed gas insulted line. In CIGRE, 2000

F. Sganzerla, J. A. A., Casagrande, and D. B. Galia. Electron are Brazila - $500 \mathrm{kV}$ and $230 \mathrm{kV}$ Compavr Lines: Design and 2. G or subconductors number in each phase, CIGRE, Paris, France, Sept.1996.

\section{Biography}

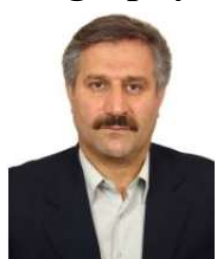

Sohrab Firouzifar was born in Damavand in Tehran, IRAN, on July 9, 1959. He graduated from The Institute of Technology of Tehran (M.Sc.), and is a $\mathrm{PhD}$ student at Malardalen University in Vasteras Sweden. He was the director of standard and quality control in Mazandaran regional electrical company in north of Iran, and is a member IEEE in IRAN, and member of the board in IEEE north of Iran. He has published several papers in Iran for example: International Power system conference (PSC) and distribution system conference about expert system in transmission substation \& power transformers utilization. He was director for technical office for 10 years, and was director $R \& D$ in M.R.E.C for 7 years. 


\begin{tabular}{|c|c|c|c|c|c|c|c|c|c|c|}
\hline \multicolumn{11}{|c|}{ Appendix: Comparison between conventional and compact line with consideration (GMD) } \\
\hline$(\mathrm{Cm}) \mathrm{GMD}$ & circuit & $\begin{array}{l}\mathrm{Cub} \\
\text { conductor }\end{array}$ & $\mathrm{C}$ & $\mathrm{X}_{\mathrm{C}}$ & $\mathrm{L}$ & $X_{L}$ & $Z_{1}$ & $\Delta u \%$ & $\frac{p}{S I L}$ & $\frac{u_{1}}{u_{2}}$ \\
\hline $70-70-140$ & 1 & 1 & 0.0001 & 30.5 & 0.01066 & 33.5 & 319 & 30 & 4.8 & 1.44 \\
\hline $20 \mathrm{KV}$ & 1 & 2 & 0.00018 & 17.5 & 0.0612 & 19.22 & 183.5 & 18 & 2.7 & 1.2 \\
\hline$"$ & 2 & 1 & 0.00023 & 14.06 & 0.04917 & 15.44 & 147 & 15 & 2.22 & 1.17 \\
\hline$"$ & 2 & 2 & 0.0004 & 7.85 & 0.02743 & 8.6 & 82.25 & 8.5 & 1.24 & 1.09 \\
\hline $70-70-70$ & 1 & 1 & 0.00011 & 29.18 & 0.10198 & 32.04 & 305 & 29 & 4.6 & 1.41 \\
\hline $20 \mathrm{KV}$ & 1 & 2 & 0.0002 & 16.19 & 0.05658 & 17.78 & 169 & 17 & 2.5 & 1.19 \\
\hline$"$ & 2 & 1 & 0.00022 & 14.59 & 0.05099 & 16.01 & 152 & 15 & 2.3 & 1.17 \\
\hline$"$ & 2 & 2 & 0.00039 & 8.09 & 0.02829 & 8.88 & 84.88 & 8.7 & 1.28 & 1.09 \\
\hline $140-140-280$ & 1 & 1 & 0.0001 & 31.17 & 0.108 & 34.22 & 326 & 16 & 4.9 & 1.2 \\
\hline $63 \mathrm{KV}$ & 1 & 2 & 0.00016 & 19.83 & 0.069 & 21.7 & 207 & 11 & 3.13 & 1.12 \\
\hline$"$ & 2 & 1 & 0.00022 & 14.3 & 0.0499 & 15.7 & 149 & 7.9 & 2.25 & 1.08 \\
\hline$"$ & 2 & 2 & 0.00036 & 8.63 & 0.03 & 9.47 & 90.4 & 4.9 & 1.36 & 1.05 \\
\hline $140-140-140$ & 1 & 1 & 0.00011 & 29.85 & 0.1043 & 32.77 & 312 & 16 & 4.7 & 1.19 \\
\hline $63 \mathrm{KV}$ & 1 & 2 & 0.00017 & 18.51 & 0.0646 & 20.32 & 193 & 10 & 2.92 & 1.11 \\
\hline$"$ & 2 & 1 & 0.00021 & 14.92 & 0.05216 & 16.38 & 159 & 8.2 & 2.35 & 1.09 \\
\hline$"$ & 2 & 2 & 0.00034 & 9.25 & 0.03234 & 10.15 & 96.98 & 5.2 & 1.46 & 1.05 \\
\hline
\end{tabular}

